\title{
Ein Fall von Schizophrenie?
}

\author{
Von \\ J. van der Torren, \\ Vervenarzt in Hilversum (Niederlande).
}

(Eingegangen am 28. September 191\%.)

Beschreibungen der eigenen Krankheit sind für die Psychiatrie noch immer wichtig; deshalb gebe ich an dieser Stelle den Auszug des Tagebuches einer 35jährigen unverheirateten Patientin, Tochter eines Verwalters einer Fabrik, das sie am Ende des Jahres 1915 angefangen hat. Die Stilfehler kann ich in der Utbersetzung nicht wiedergeben; sie bleiben aber innerhalb der Grenzen der Erziehung und Bildung der Patientin, wie auch das Fehlen der Interpunktion an mancher Stelle. Wo es mir erwünscht erscheint, gebe ich in der Úbersetzung die eigenen Worte der Patientin.

Auszug: Pat. fängt an mit Jugenderinnerungen, die Beschreibung der elter'lichen Wohnung und dic Schuljahre betreffend. Die Eltern lebten etwas über ihren Stand, hatten viele Bekannte und vielen Besuch, deshalb große Ausgaben, und verarmten. Pat., die früher Klavierstunden bekam, mußte jetzt im Alter von vierzehn Jahren eine Stelle suchen, was sie sehr enttäuschte. Wegen Krankheit der Mutter kehrte sie später wieder zur Hilfe in das elterliche Haus zurück. Im Alter von 18 Jahren verlobte sie sich; die wahre Liebe, schreibt sie, ist dies aber nicht gewesen, denn jetzt, nach 15 Jahren, weiß ich nicht mehr, wie er aussah, und kommt er mir niemals in meine Gedanken. Nach einem Jahre wurde die Verlobung denn auch aufgehoben. Zwar ist es eine angenehme Zeit, das Alter von 18 Jahren, man sieht das Leben wie ein Luftschloß, aber später entdeckt man, daß nur sehr wenig davon übrigbleibt, ausgenommen vielleicht für einige Auserwählte. Die Jahre gingen vorüber, das eine wie das andere. Eines Tages aber (im Alter von gut 20 Jahren) geschah etwas Besonderes, das ihrem Leben eine andere Wendung gab, und das sie später viele Tränen weinen ließ. Bei der häuslichen Arbeit wurde ihre Aufmerksamkeit auf einen Herrn in einem Hause auf der anderen Seite der Straße gelenkt und weil sie gut 20 Jahre alt war, war es cine natürliche Sache - meint sie - daß sie ihn ansah. Sie fand ihn hübsch. Der Herr guckte auch, und dieses Spiel wiederholte sich die rächste Zeit immer wieder. Der Herr, schreibt sie, war ein Sonderling, und vielleicht war auch dies eine der Ursachen, daß sie sich in ihn verliebte. Er liebte dic Natur, seine Fenster waren immer offen, auch nachts oder wenn es fror, daß die Bäume krachten. Später ahmte sie dies nach. Auch steht er nur mit dem Oberhemd bekleidet auf dem Balkon, und daß er sich dabei für die kleinlichen und engherzigen Meinungen der Menschen gleichgültig zeigte, liebte sie an ihm. Er hatte eine männliche Gestalt, war breitschultrig und doch geschmeidig wie ein Sportsmann. „Nun ist es zwar dumm, wenn eine Frau meint, ein Mann sei verliebt in sie, wenigstens wenn 
sie einander nie gesprochen haben, aber ich hoffte es, und deshalb dachte ich es mir auch so. Ich fing an, ihn außerordentlich stark zu lieben, sein Bild kam mir niemals aus den Gedanken, sogar nicht in der Nacht. Die Sache kam jedoch nicht weiter, ich wurde verdrießlich, deprimiert, reizbar, vernachlässigte die Arbeit, suchte Trost in der Religion, flehte Gott, aber vergebens, an, mit diesem Manne doch noch einmal vereinigt zu werden, wurde krankhaft und hinfällig. Wer nicht selbst so etwas erfahren hat, ist nicht imstande, solch einen Seelenzustand zu verstehen. Endlich wurde ich geisteskrank. In diesem Zustande stellte ich mir fortwährend vor, mit ihm verheiratet zu sein und fühlte mich außerordentlich glücklich. Ich dachte mir sogar ein Töchterchen von etwa vier Jahren (Pat. war damals 24 Jahre alt - Ref.) zu haben, mit dem ich spazieren ging." Pat. erzählte schon, sie suchte Trost in der Religion, und betete viel zu Gott, und nun erzählt sie, daß in dieser Periode der Geisteskrankheit etwas ganz Übernatürliches geschah. Sie wundert sich darüber jetzt immer noch, und meint, daß einer, der später ihr Tagebuch liest, meinen wird, sie sei noch immer nervenkrank, und doch sei sie jetzt gesund. Daß sie die Wahrheit erzähle, würde sie gerne beschwören. Und dieses Ửbernatürliche besteht darin, daß sie Gott sah. ,Nicht im Traum oder wie eine Vision, sondern echt, wie ich einen anderen Menschen oder mich selbst sehe. Er hatte einen langen weißen Bart und redete mit mir. Gott ließ mich arbeiten. Ich durfte nicht mit meinen Eltern reden, auch nicht mit anderen Leuten, durfte nicht essen, mußte im Korridor auf und $a b$ gehen und mich dort vor einen Geist hinstellen und diesen mit starkem Blick ansehen. Die Bedeutung dieser Dinge ist mir nie klar geworden." Einige Tage hielt die Pat. es aus, nicht zu essen, aber leider wurde die Versuchung zu stark, wie auch der Hunger, sie wurde ungehorsam und widerstrebte nicht länger der Zwangsfütterung. Wohl hätte sie gerne sterben wollen, aber der Tod eines Märtyrers fiel ihr zu schwer. Unter der Zwangsfütterung kehrten die Kräfte und darauf auch die Gesundheit zurück und Pat. kam wieder nach Hause. Später, zu Haúse, sprach Gott wieder zu ihr und sagte: "Ich gehe weg, Kleine." Diese Beschreibung ihrer Erlebnisse während ihrer Krankheit, sie schwört es im Namen Gottes und aller Menschen, ist die Wahrheit; mehr Beweise beizubringen zur Überzeugung anderer, dazu ist sie nicht imstande.

Doch war ihre Liebe noch immer da, hatte sogar eher zugenommen. Den betreffenden Herrn sah sie einige Male wieder, auch in der Kirche, obgleich sie nicht wußte, daß er kirchlich gesinnt war. Acht Jahre lang blieb sie gesund, obgleich auch während dieser ruhigen Zeit zu Hause, wie auch jetzt noch, die Geister dann und wann mit ihr reden, und sie überzeugt ist, daß dies keine Halluzinationen sind. Daß es Geister in der Welt gibt, versucht sie mit Hilfe theosophischer Lehrsätze zu beweisen.

Nach achtjähriger Gesundheit erkrankte sie wieder in gleicher Weise. Sie stand wieder in dem Wahn, schreibt sie, verheiratet zu sein und Kinder zu haben, aber später sah sie ein, daß dies Unsinn war. Sie bekam wieder Visionen. ,Ich sah den Himmel, oder vielleicht auch die neue Erde, das weiß ich nicht bestimmt, herrlich schön. Es wohnten Menschen dort, Ehepaare; sie waren recht schön und glücklich wie Kinder. Auch gab es prächtige Gärten. Später sah ich den Teufel, einen Geist mit schwarzem Bart und fürchtete mich sehr. Der Teufel saß am Tisch, und der Geist eines Mädchens am Oberende des Tisches. Es war abscheulich. Später legte der Teufel sich in eines der Betten mir gegenüber und er erzählte mir, daß in der Nacht einer ermordet werden müsse, Der Krankensaal glich einem Zimmer, ich sah mehrere Geister, unter welchen einer mit schwarzen Locken, aber auch sehr häßliche, waren. Ich fürchtete mich zu Tode und schrie aus aller Macht ,Gottie', und schrie immer wieder. Der Arzt kam und gab mir eine Injektion. Die Nacht war entsetzlich; ich glaube, dies nicht deutlich machen zu 
können. Ich hörte ein heftiges Gewitter, nicht wie gewöhnlich, sondern wie zehn Gewitter zusammen. Darauf verging die Welt und der Mensch war geschieden von Gott und fühlte sich grenzenlos unglückselig. Als am nächsten Morgen das Bewußtsein wieder zurückkehrte, fragte ich die anderen Pat., ob sie es auch gehört hatten, aber sie lachten mich aus. Die Genesung trat ein, ich bekam Freundinnen in der Anstalt, und falsche Scham hielt mich davon zurück, ihnen gegenüber bei der Meinung zu bleiben, daß diese Gesichter wirklich gewesen sind; ich fing an zu heucheln, aus Furcht ausgelacht zu werden. Im Anfang dieser zweiten Erkrankung, als ich noch zu Hause war, hat der Herr Jesus auch mit mir geredet. Er gab mir schöne Visionen, u. a. die Keimung des Samens im Erdboden; es war außerordentlich schön. Immer wieder aber wollte ich den Teufel kennenlernen, und eines Abends kamen zwei Männer, einer mit schwarzen Augen, die mich auf einen Tragstuhl legten. Ich schrie: ,Der Teufel, der Teufel!" und meinte, daß er mich zur Hölle bringe. Auch habe ich das Reich der Finsternis gesehen. Der Teufel sagte mir, daß, wenn ein Mensch stürbe, drei seines Reiches versuchten, die Seele zu zerstören. Es war ein großes, mächtiges Reich mit vielen Seelen oder unreinen Geistern. Ich schreibe alles auf, wie es mir geoffenbart worden ist; zwar wei $ß$ ich nicht, ob Gott dies erlaubt, aber es ist kein Mensch, mit dem ich über meine geistigen Gesichter reden kann; denn sie würden sagen, ich sei geisteskrank oder sie würden es nicht verstehen. Da entschloß ich mich, es so gut wie nur irgendwie möglich aufzuschreiben - wird man es je verstehen? Wie ich schon sagte, mein Verstand kehrte wieder zurück, aber mein Gewissen ließ mir keine Ruhe, denn ich hatte Gott nicht gehorcht und doch hatte der Gehorsam meine Kräfte ein wenig überstiegen. Ich betete immer, ob ich es wieder versuchen dürfte, und endlich wurde mein Gebet erhört. Auch sah ich Engel in weißen Gewändern, und ein großes Feuer, die Hölle, und der Teufel wurde von Jesu mit einer Geißel ins Feuer geschlagen. Weshalb ich diese Visionen empfangen habe, weiß ich nicht, Gott hat mir dies nicht geoffenbart, ebensowenig wie ihre Bedeutung. Besser wie ein anderer Mensch bin ich ja nicht, öfter unartig, aber wohl habe ich außerordentlich viel gebetet und viel an Gott gedacht.

Wieder erschien Gott mir, ich dürfte nichts essen, reden oder schlafen, aber wieder wurde mir eines Tages, während ich im Bade war, die Versuchung zu stark und ich aß wieder. Gott verbot mir, Fleisch zu essen, aber gerade Fleisch liebe ich so sehr. Infolge der Nahrung kehrten meine Kräfte wieder, ich genas und kam wieder nach Hause. Bei der Abreise aus der Irrenanstalt stand eine wirkliche Gestalt an der Türe mit dunklem Antlitz und lauerndem Blicke; sie ist dort zurückgeblieben und als ich fortfuhr, stand sie hinter der gläsernen Tür. Ich denke, der Teufel? Vielleicht ist dies eine Strafe Gottes, denn immer stand er an der gleichen Stelle. Ich möchte gerne wissen, ob er noch immer da ist. Gott selbst ist cine majestätische Erscheinung mit langem, weißem Bart und durchdringendem Blick, aber nicht zum Fürchten. Er ist einfach, ungekünstelt, wie Christus auch. Ich meine, Gott beschäftigt sich wohl nicht mit einem bestimmten Glauben, denn als er mir zum ersten Male erschien, war ich Remonstrant, also einem mehr modernen Glauben zugetan. Ich meine, es kommt nur darauf an, wie die Menschen eigentlich sind und handeln, und auch, wenn die Menschen mehr mit ganzer Seele beteten, würden sie auch wohl hier auf der Erde schon in Gemeinschaft mit Gott leben. Aber dies ist, es spricht für sich, nur ein Gedanke von mir und nicht absolut sicher. -

Meinen früheren Nachbarn habe ich noch immer nicht vergessen, und ich werde ihn wohl nie vergessen, sonst hätte ich nicht so viel und stark durch ihn gelitten, aber zum Glück werde ich ihn an meinem neuen Wohnort wohl nicht begegnen und deshalb wird die Wunde auch nicht mehr aufgerissen." Später verlobt sich ihre jüngere Schwester, und Pat. wundert sich darüber, daß dem einen 
Menschen nur Glück beschert ist und dem anderen nur Unglück. Sie denkt daran, $\mathrm{da} B$ sie es selber vielleicht auch suchen muß. Aber wie? Denn ihr Leben ist so einsam und sie liebt nicht die Menschen. 10. Mai 1917 schreibt sie: „Was ist das doch abscheulich, den ganzen langen Tag denke ich an ihn, gestern auch. Ist denn dagegen nichts zu tun? Einen anderen Mann heiraten? Aber wenn ich in diesem Fall dann nur keine Reue bekomme. Ich weiß selber nicht, wo ich suchen muß. Bei Gott? Aber der tröstet mich auch nicht. Viel Zerstreuung suchen? Aber wie? Die Theosophie bietet einen besseren Trost, sie sagt, daß die Seelen doch am Ende miteinander vereinigt werden. Die Bibel jedoch sagt: Im Himmel wird weder zur the gegeben, noch zur Ehe genommen, sondern man lebt wie Engel. Doch weshalb soll ich ein Verlangen zum Himmel hegen, denn ohne ihn ist auch der Himmel mir gleichgültig. Es ist und bleibt ein fürchterliches Leiden; vielleicht endet es niemals. Ich möchte so gerne glücklich sein mit ihm. Es ist ein törichter Gedanke, der mir im Kopfe herumspukt. Irgendwo auf dem Lande mit ihm zusammen wohnen in einem schönen heimeligen Landhause mit Garten. Ich würde mich hübsch kleiden, öfter in weiße Kleider, denn das ist gesellig; und auch einfache hübsche Möbel. Dann und wann mal ein paar Bekannte zu Besuch empfangen. Ach, welch eine Torheit, vielleicht hat er sich schon längst verheiratet und hat auch Kinder. Darum habe ich zu Gott nun so oft gebetet, es hat aber gar nichts geholfen. Warum doch nicht? ??" -

Ich lasse jetzt noch drei lyrische Gedichte der Patientin folgen, welche vielleicht nicht ohne Kunstwert sind, jedenfalls aber ihre Stimmung schön illustrieren.

I.

1. Ein ruheloses Verlangen treibt mich fort

Und führt mich auf vielerlei Wegen.

Einmal ist's „das Gute", das mich entzückt,

Dann wieder ist's , die Welt", die mir entgegenlacht.

Und wie ich auch kämpfe oder bitterlich zuweilen spotte,

Doch lenkt der Wille unseres Vaters das Los.

2. Ach, zuweilen ist das Leben mir eine Hölle,

Verzehrt mich ein endloses Verlangen,

Darauf wieder scheint kein Frieden zu bestehen,

Und klopft mir das Herz so bange.

So zwecklos, so einsam scheint dann mir mein Pfad,

Als ob ich weder Freude noch Auskunft mehr hätte.

3. Was macht mich so traurig und schwermütig,

Wie scheint mir das Leben so düster,

So manchmal lacht die Welt mir entgegen,

Aber sie zerstört auch das Gute.

Dann ist es ein Kampf zwischen Welt und Gott,

Doch lenkt er, uncerem Zweifel zum Trotz, unser Los.

4. Ein Mysterium ist das Leben, unsicher der Weg,

Öfter stürmen vielerlei Fragen.

Sind Mut und Zuversicht so weit entfernt.

Aber was nützt uns das zwecklose Klagen,

Wir müssen immer weiter, bergaufwärts führt der Pfad,

Der ausläuft vielleicht auf ,Die Ewige Stadt". - 
II. Neujahrsnacht.

1. Das alte Jahr ist vorübergegangen, Mit seiner Freude, seinem Leide.

Es ließ mir viele Fragen übrig,

Und aus meiner Seele steigt der Schrei empor:

o Gott, gib mir die Freude wieder

Der Jahre, welche vorübergegangen sind.

Sie sind alle rasch entflohen,

Und ließen mir meinen Schmerz zurück.

2. Die Einsamkeit macht mich so bange,

Führt Angst und Zweifel in mein Herz hinein.

Das müde Haupt, es wünscht schon lange

Zu ruhen wegen der großen Schmerzen.

Glück, wo findet man's auf Erden,

Für mich, ich meine, besteht es nicht.

Meine Augen sind schon müde vom Stieren,

Aber nirgendwo sehen sie den Frieden.

3. Die Zeit, sie rollt so ruhelos fort,

Und schleppt alles mit sich fort.

Nichts gibt es, das mich entzückt.

Woher stammt doch all dieses Herzeleid?

Aber sieh, es gibt doch auch noch Blumen,

Recht feine blühen auf meinem Pfade,

Und manche Rose auch blühte auf,

Welche ich sehr geliebt habe.

4. Doch ist das Herz so leer und fremdartig,

Es sehnt sich jedesmal wieder nach mehr,

Es sucht und wendet sich seufzend ab,

Und denkt über frühere Zeiten.

Aber doch sehe ich wieder einen Stern stehn,

Ganz hoch dort oben am Himmel.

Ich werde nur langsam weiter gehn,

Während meine Seele leicht aufseufzt. -

(31. Dez. 1916.)

III.

1. Wenn die Lebensstürme kommen,

Und die Angst das Herz berührt,

Wenn das Leben kalt und trübe,

Und uns alle Freud' verläßt,

Dann steigt aus der Seele empor

Ein Gebet zu dir, o Gott,

Laß das müde Haupt doch ruhen,

Gib uns Frieden mit unserem Los.

2. Wenn wir anderen Leuten begegnen,

Denen das Leben alles bietet,

Wenn ihnen in ihrem frohen Leben

Das Glück aus den Augen strahlt,

Ja, dann steigt die bitt're Klage

Aus unserem Herzen gen Himmel empor,

Weshalb müssen wir so leiden,

Weshalb hat uns das Leben betrogen. 
2. Wenn die Nebel nicht weichen wollen, Und wir öfter schreien vor Leid,

Wenn die Fragen uns entgegen stürmen, Und nicht ruhen vor unserem Gebet, $\mathrm{Ja}$, dann ist im Herzen

Soviel Unruhe und Leid, Daß nur das Grab mir zulacht,

Weil ich nirgendswo Auskunft sehe.

Wer viel gelitten hat,

Und nirgendswo die Ruh' gefunden,

Der wird diese Verse verstehn,

Weil sie dem Leide entsprangen.

Wie die Mutter erzählte, ist Pat. leicht gereizt, sie ist bald böse und sehr übelnehmend. Sie nimmt es sehr übel, wenn man ihre Behauptungen nicht ernst nimmt oder auf ihre frühere Krankheit hindeutet. Pat. stimmt dem zu und fürchtet sich auch, daß die Mutter sie wieder in die Irrenanstalt wird aufnehmen lassen. Auch ihr Bruder ist nervös und dies verursacht zuweilen häusliche Streitigkeiten. Pat. hat denn auch vor einigen Tagen mit einem Messer gedroht und einem der Familienmitglieder angeboten, ihm die Kanne des Waschbeckens an den Kopf zu werfen.

Bei meinem Besuch ist sie ruhig und erzählt mir alsbald von ihrer hoffnungslosen Liebe, obgleich sie kurz vorher gesagt hatte, daß sie das nie einem anderen Menschen erzählt habe oder erzählen werde. In Wirklichkeit weiß auch ihre Mutter davon. Trost hat sie gesucht in der Religion, aber auch da hat sie die Lösung für ihren Konflikt noch immer nicht gefunden, sie sucht noch jetzt bei der Theosophie, der Lehre Tolstois, dem Spiritismus, usw. und betrachtet sich selber offenbar als einen philosophischen Geist, obgleich sie auch sagt, nicht mehr zu sein als andere Menschen und in Wirklichkeit von den elementaren philosophischen Dingen nicht viel versteht. Für die zwei akuten Phasen ihrer Geisteskrankheit zeigt sie eire gewisse Krankheitseinsicht; gibt auch zu, krank gewesen zu sein, ist dagegen vom wirklichen Bestehen der Geister überzeugt, erklärt dies mit theosophischen Lehrsätzen und sieht sie auch in ihren ruhigen Tagen dann und wann wieder. Obgleich sie den Haushalt besorgt, interessiert dieser sie nur wenig; denn die Wichtigkeit des gewöhnlichen Lebens und der täglichen Arbeit verschwindet ihr gegenüber der Ewigkeit und dem Leben nach diesem irdischen Leben. -

Und die Diagnose? Wie den Äußerungen der Patientin zu entnehmen ist, hat man bei ihrer ersten Aufnahme in die Anstalt an Hysterie gedacht. Während die Kranke sich in der Zelle befand und sich nackt ausgekleidet hatte, war der Arzt gekommen und hatte einem Kollegen gegenüber gesagt: diese Patientin leide an Hysterie. Sie hatte sich darüber sehr entrüstet und den Arzt gefragt, was er damit meine, denn sie glaubte, daß der Name Hysterie so ungefähr gleichbedeutend sei mit einem in sexueller Hinsicht unmoralischen Verhalten. Zwar hatte sie sich nackt ausgekleidet, fügt sie hinzu, aber das kam daher, daß sie eine Stimme aus dem Himmel hörte, die ihr befahl, sich nackt auszukleiden, damit sie in den Himmel aufgenommen werden könnte. 
Wie nahe damals, mit der zur Verfügung stehenden Anamnese, diese Diagnose nun auch lag, so läßt sich die Hysterie jetzt wohl ausschließen. Es liegt doch eine chronische Psychose vor, in deren Verlauf, mit einer Zwischenzeit von acht Jahren, zwei akute Schübe aufgetreten sind, für welche nur mangelhafte Krankheitseinsicht besteht, während in dieser Zwischenzeit und auch jetzt noch dann und wann immer wieder Halluzinationen (Geister) auftreten, für welche Erscheinungen jede Krankheitseinsicht fehlt und für welche Patientin in theosophischen Lehrsätzen eine Erklärung sucht.

Obgleich eine exquisit depressive Betonung der Lebenserfahrungen besteht (siehe die drei Gedichte) läßt auch das manisch-depressive Irresein sich wohl ohne weiteres ausschließen.

Gleiches gilt für die chronische Paranoia, denn der Anfang war akut und zur Bildung eines ausgesprochenen Beziehungs-, Verfolgungs- und Größenwahns ist die Psychose ebensowenig fortgeschritten als zu einer Systematisierung solcher Wahnideen.

Auch die Kraepelinsche Dementia praecox läßt sich meines Erachtens ausschließen, wenn man wenigstens die Grenzen dieser Krankheit nicht bis ins Unermeßliche ausdehnen will. Obgleich die Patientin schon vor elf Jahren erkrankt ist, ist es zum typischen Verhalten eines Praecoxkranken gar nicht gekommen und kann von einer Demenz weder in intellektueller noch in affektiver Hinsicht die Rede sein.

Wie so oft gelingt auch in diesem Fall die Unterbringung im System nicht recht. Úbereinstimmung besteht mit Wernickes Residuärsymptomen und residuärer Halluzinose ${ }^{1}$ ), aber auch damit gelangt man nicht zu einer richtigen Diagnose. Auch mit der circumscripten Autopsychose auf dem Boden der überwertigen Idee stimmt der Verlauf wohl nicht.

Dementia praecox oder Residuärsymptom, mit diesen beiden Namen tritt auch der gewaltige Unterschied zwischen Wernicke und Kraepelin und zwischen den Lehrbüchern dieser beiden Autoren hervor. Zu einer Einteilung bestimmter scharf umschriebener Krankheiten in einem System ist Wernicke nicht gelangt, denn er wußte, daß dies mit den damaligen Kenntnissen (und das gilt auch jetzt wohl noch in mancher Hinsicht) unmöglich war. Sogar die progressive Paralyse ist ihm nur eine ätiologische Zusammenfassung von sonst untereinander sehr verschiedenen Psychosen ${ }^{2}$ ).

Anders Krae pelin. Auch ihm ist es wohl klar, daß eine definitive Umgrenzung verschiedener Krankheiten heute unmöglich ist. Kommt nun auch die Praxis in vielen Fällen ohne Diagnose einer bestimmten Krankheit aus, ein praktisches System kann auch ihr nicht gleich-

1) Lehrbuch. Zweite Aufl. S. 113 und 154.

2) Ibid. S. 458. 
gültig sein, und überdies drängt die Wissenschaft zu einer UUbersicht und einer wissenschaftlichen Zusammenfassung der Erfahrungen. Da hat nun K rae pelin sein System gegeben, sah sich aber beim Anwachsen der Erfahrungen immer wieder genötigt es abzuändern, Grenzen früherer Krankheiten auszudehnen oder einzuschränken, neue Krankheiten aufzustellen, um es immer wieder von neuem zu versuchen, der Psychiatrie ein schönes und praktisches Haus zu bauen. Und jeder Psychiater wünscht Mitarbeiter zu sein; zum Glück kommt es aber nicht weiter, und kann es auch nie weiter kommen als zu einem Turme Babels, wobei sogar die Verwirrung der Sprachen nicht ausbleibt. Und heute scheinen wir wieder zur Einsicht zu gelangen, daß es eigentlich weit besser ist, den Versuch der Darstellung eines Gebäudes der Zukunft zu überlassen und uns mit der Aufstellung neuer Krankheitstypen zu begnügen. Unter Voraussetzung vorangehender Zeilen könnte man jetzt meinen, unsere Kranke wäre am besten bei der Schizophrenie Ble ule rs unterzubringen, besonders da Bleuler selber schreibt, daß er das Wort Schizophrenie nur der Bequemlichkeit wegen im Singular gebrauche, obschon die Gruppe wahrscheinlich mehrere Krankheiten umfasse.

In einer der letzten Nummern dieser Zeitschrift versucht nun Bornstein $^{1}$ ) die durch die psychische Spaltung charakterisierte Schizophrenie Bleulers in drei Untergruppen einzuteilen: 1. die Schizothymia reactiva, welcher Krankheitstypus dadurch zustande kommen soll, daß sich ein einziger, vorwiegend mit einem wirklichen Erlebnis verbundener Komplex von der gesamten Psychik abspaltet und somit meistens eine Reaktion gegen jenes Erlebnis darstellt. Das klinische Bild besteht in direkter Verwirklichung des in diesem Komplex enthaltenen, im Leben aber unerfüllt gebliebenen Verlangens. Dieser Typus von Persönlichkeitsspaltung weist weder Assoziationsstörungen, tiefgreifendere Affektivitätșänderungen, Verfolgungsideen, noch Halluzinationen auf; trotzdem hinterläßt er eine andauernde Umwandlung der Persönlichkeit, wobei gleichzeitig eine allgemeine Besserung im praktischen Lebenssinne möglich ist und sehr häufig vorkommt.

2. Die Schizophrenie (Bleuler). Vermag ebenso wie Typus I als Reaktion auf ein schweres wirkliches Erleben zu entstehen, kann aber unabhängig von den Lebensangelegenheiten spontan zum Ausbruch kommen, als ein Schub des zugrunde liegenden Prozesses. Vom ersten Typus unterscheidet sie sich durch spezifische Assoziationsstörungen, meistens primäre affektive Verkümmerung oder Disproportion der Affekte und der Vorstellungen, durch das Auftreten von Verfolgungsideen und Halluzinationen. Dieser Typus führt meistens

$\left.{ }^{1}\right)$ M. Bornstein, Über einen eigenartigen Typus der psychischen Spaltung (Schizothymia reactiva) 39, 86. 1917. 
eine dauernde Umwandlung der Persönlichkeit herbei, doch kann derselbe auch mehrmals im Leben des Individuums wiederkehren, ohne zu endgültigem Blödsinn zu führen; im Gegenteil, er kann jedesmal in gutartiger Weise verlaufen, sehr lange und gute, einer völligen Genesung gleichkommende Remissionen geben. Je nach dem Utberwiegen dieser oder jener Symptome, lassen sich verschiedene Abarten dieses Typus unterscheiden (Schizophr. katatonica, paranoides usw.).

3. Dementia schizophrenica, Fälle von psychischer Spaltung umfassend, mit progressivem Verlauf und endigend in schwerem Blödsinn spezifischen Charakters.

UUbergänge zwischen diesen drei Typen kommen, wie Bornstein meint, vor.

Sind wir nun imstande, unseren Fall bei einer dieser drei Untergruppen der Schizophrenie unterzubringen, oder stellt er einen Übergangsfall dar? Soweit wir die Krankengeschichte bis auf heute überblicken, scheidet der Typus der Dementia schizophrenica von vornherein aus. Utberdies ist es nach Bornstein unzweifelhaft, daß ein Teil der zum zweiten Typus hinzugezählten Fälle sogar nach mehrmaligen Remissionen endgültig in Blödsinn übergehen kann. Aber auch der zweite Typus, die Schizophrenie Bleulers in engerem Sinne stimmt nicht. Zwar sind im Verlauf viele Halluzinationen aufgetreten, zwar treten solche dann und wann noch immer auf, es fehlen aber die Verfolgungsideen wenigstens in ausgesprochenem Maße, und besonders, es fehlt die Verkümmerung oder Disproportion der Affekte zu den Vorstellungen. Der Typus der Schizothymie dann? Aber es treten in unserem Fall massenhafte Halluzinationen auf. Also ein thergangstypus? Sehen wir aber die zwei Fälle Bornsteins näher an, so erweist es sich, daß in seinem ersten Fall im Anfang einige Halluzinationen (vielleicht auch Pseudohalluzinationen), auftraten, während es sich in seinem zweiten Falle zweifellos, wie er sagt, um dlusionen und nicht um Halluzinationen handelte. Abgesehen von der Frage der Pseudohalluzinationen will es mir nun doch vorkommen, daß Bornstein Illusionen und Halluzinationen zu scharf voneinander zu trennen sucht. Wissen wir doch, daß es hinsichtlich des Wesens dieser krankhaften Erscheinungen nicht an erster Stelle darauf ankommt, ob wirkliche, äußeren Reizen entspringende Empfindungen da sind oder nicht, sondern daß die Grundursachen im Auftreten beider pathologischen Symptome zentral in krankhaften Veränderungen des Vorstellungs- und Gefühlslebens des Patienten zu suchen sind.

Also die Schizothymie? Nun, auch dies ist nicht ohne weiteres zuzugestehen. Zwar liegt sowohl in meinem Fall wie in den beiden Fällen Bornsteins die sogenannte Flucht in die Krankheit auf der Hand, es bilden aber die drei Typen Bornsteins Untergruppen einer mehr 
umfassenden Schizophrenie, welch letztere dadurch gekennzeichnet ist, daß während der Krankheit eine S palt ung der vorher nor malen Persönlichkeit auftritt. Seine eigenen Fälle der Schizothymie sind dann wieder dadurch gekennzeichnet, daß ein spezifischer Typus psychischer Spaltung besteht, d. h. ein psychischer Prozeß im Jas perschen Sinne, „dessen Spezifität" in folgenden Merkmalen besteht: 1. Er stellt einen ",psychischen Proze $\beta^{\prime \prime}$ dar, der eine zweifellose Re aktion gegen ein bestimmtes Erlebnis ist; er entsteht im unmittelbaren Zusammenhange mit diesem Erlebnis, und der Inhalt der Psychose entspricht ihm genau. So haben wir es also mit einem reaktiven psychischen Prozeß zu tun.

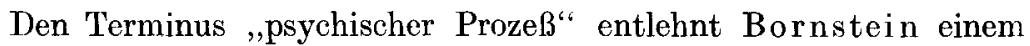
Aufsatze Jaspers"). Worin besteht nun das Wesen des ,,psychischen Prozesses?" "Prozesse", sagt Jaspers, ,sind unheilbare, der bisherigen Persönlichkeit heterogene Veränderungen des psychischen Lebens, die entweder einmal oder isoliert, oder wiederholt und allgemein und in allen Ưbergängen zwischen diesen Möglichkeiten in dasselbe eingreifen" (S. 608). Dies im Gegensatz zur krankhaften Entwicklung einer Persönlichkeit. „Wenn jetzt allgemein von der Entwicklung einer Persönlichkeit gesprochen wird, so kann das eben nur heißen, daß wir die Vorgänge, die aus irgendwelchen Gründen krankhaft genannt werden, in diesem Falle aus dem Ineinanderspiel der psychologischen und rationalen Zusammenhänge, die eingebettet sind in einen bei aller Disharmonie und Haltlosigkeit doch einheitlichen, ursprünglich angelegten, objektivierten psychischen Entwicklungszusammenhang verstehen oder erklären können 2)." Die Prozesse führen zu einer „dauernden unheilbaren Veränderung". Gradweise Unterschiede zwischen Prozessen und Entwicklungen einer Persönlichkeit gibt aber auch Jaspers zu (S. 637). Aus der Erwägung heraus, daß jede Lebensentwicklung ein Prozeß ist, in den einfühlbare und rationale Zusammenhänge eingebettet sind, daß aber der „Prozeß“ des normalen Lebens als „Entwicklung" aufgefaßt werden kann, insofern man intuitiv in ihm die Einheit der Persönlichkeit erfaßt; aus der Erwägung auch der hochgradigen Subjektivität dieser Intuition heraus läßt Jaspers Ubergänge zwischen dem „Nenen“, das als der Persönlichkeitseinheit eigentümlich in bestimmten Lebensphasen auftritt, und dem „Neuen“, das ihr als Heterogenes gegenübertritt, zu. In der Lebensentwicklung sind also einfühlbare und rationale Zusammenhänge eingebettet; restlos verstehen oder erklären können wir aber auch diese Entwicklung nicht. Ubergangsfälle führt Jas pers selber an.

1) K. Jaspers, Eifersuchtswahn. Ein Beitrag zur Frage: „Entwicklung einer Persönlichkeit“ oder „ProzeB“. Diese Zeitschr. 1, 567. 1910.

2) Ibid. S. 606 .

Z. f. d. g. Neur. u. Psych. o. XXXIX. 
Sehen wir uns nun wieder genauer erstens die zwei Fälle eines Prozesses Jaspers' an, so tritt im ersten Fall wirklich an einem bestimmten Zeitpunkt seiner Entwicklung etwas Neues auf, das weder rational, noch einfühlbar an ein Ereignis in verständlicher Weise sich anschließt, noch sich aus der Persönlichkeit verstehen läßt. Dies jedoch unter der Einschränkung, und ich möchte das mit Nachdruck betonen, daß die persönliche Entwicklung des Kranken bis zum genannten Zeitpunkte nicht restlos bekannt ist (und dies wird nie und in keinem Fall möglich sein), so daß immer die Möglichkeit bestehen bleibt, daß bei besserer Kenntnis dieser persönlichen Entwicklung dasjenige, was uns jetzt als etwas „Neues" und „Heterogenes" erscheint, verstanden werden könnte.

Beim zweiten Patienten kann von solch einem Zeitpunkte und von der Entwicklung einer systematischen Wahnbildung innerhalb „einer relativ kurzen Spanne Zeit, die sich nach keiner Seite hin scharf abgrenzen läßt, jedenfalls im Laufe eines Jahres", meines Erachtens doch nicht gut die Rede sein, man müßte denn annehmen, daß die Aussage des Patienten, ,er habe alle diese Ungeheuerlichkeiten lange still ertragen", und die Frau habe ihm ,das Leben von Anfang an zur Hölle gemacht", nur Erinnerungsfälschungen sind, zu welcher Annahme man nicht ohne weiteres berechtigt ist. Was da doch in jedem Menschen vorgehen kann und vorgeht, was seinen nächsten Angehörigen, mit denen er jahrelang zusammenlebt, gänzlich verborgen bleibt!

Und nun die zwei Fälle Bornsteins. Jaspers weist auf die hochgradige Subjektivität der intuitiven Erfassung der Persönlichkeitsentwicklung hin, und dies ist im Auge zu behalten. Ich kann mir aber mit dem besten Willen nicht vorstellen, weshalb es bei der ersten Patientin etwas „Neues“ darstellt, daß es ihr einmal so vorkam, ,daß ihr Bruder und Vater, am Fenster stehend, sich darüber unterhielten, daß G. in W. sei, aber doch würden sie ihn nicht ins Haus hineinlassen “ (S. 106). Meines Erachtens läßt sich dieses „Gefühl", das Patientin hatte, ganz aus der Persönlichkeit und dem Vorleben der Patientin verstehen, und könnte auch ein Normaler unter solchen Verhältnissen solch ein „Gefühl“ bekommen. Und gleiches gilt für die zweite Patientin; auch bei ihr können wir es verstehen, wie aus Wunscherregungen das vom Verfasser vorausgesetzte und deduzierte krankhafte Urteil der Kranken, ihr Mann und ihre Kinder seien noch am Leben, hervorquillt. Ich sage, wir können es verstehen, nachfühlen, soweit wir eben Psychisches verstehen und nachfühlen können, denn restlos erklären oder auch nur verstehen können wir nie, sogar beim Normalen nicht, geschweige denn beim Kranken.

In diesem Sinne aber können wir sogar viele der religiösen Wahn- 
ideen der zweiten Patientin Bornsteins verstehen, wo sie z. B. sagt (S. 131): „Wir haben Messiaszeiten erlebt; als sie Kind war, prophezeite ihr Großvater, daß sie Messiaszeiten erleben wird, daß sich Waggons, Tramwagen ohne Pferde bewegen werden"; wenn es wenigstens erlaubt ist, Parallele zu ziehen zwischen den Erwartungen, welche in gewissen Volkskreisen in den Niederlanden sowie auch offenbar in Polen leben.

Meines Erachtens ist es nun auch in unserem Fall nicht notwendig, das Auftreten eines Prozesses an einem bestimmten Zeitpunkt anzunehmen zur Erklärung des Verlaufs. Soweit das immerhin möglich ist, tritt das Einfühlbare in der Entwicklung der Krankheit deutlich zutage: zuerst die Flucht in die Religion, wenn ich es so nennen darf, wobei die Patientin Trost sucht, und wenn sie auch dort nicht die Lösung findet, die Flucht in die Krankheit, und noch später sucht sie die Lösung in philosophischen Systemen usw. Daß dies alles in der Psychose in krankhafter Weise verarbeitet und zusammengefügt wird, liegt auf der Hand.

Und woraus sollte auch solch ein psychischer Prozeß entstehen? Jas pers sucht die Ursache des Auftretens eines psychischen Prozesses in dem Auftreten zu einer bestimmten Zeit eines physischen Prozesses in den direkten, höchst komplizierten physischen Parallelvorgängen des psychischen Geschehens, und weist dazu hin auf die Entwicklurg der Tumoren aus der Anlage heraus. Dabei bleibt aber auch die Ursache dieses Auftretens eines physischen Parallelprozesses dunkel. Weiter können wir vielleicht kommen und tiefer vordringen, wenn wir, wie ich das zu erläutern versuchte, annehmen, daß solch ein psychischer Prozeß nicht etwas absolut Neues darstellt, sondern daß Zusammenhänge, und sogar einfühlbare Zusammenhänge mit dem vorangehenden, noch normalen psychischen Zustand bestehen. Was mir dann in meinem Falle und in anderen Fällen gleicher Art als etwas Besonderes auffällt, ist die außerordentlich lange Nachwirkung des Affektes. Im Gegensatz zur normalen Psyche kehrt bei unserer Kranken der Affekt nie zur normalen Ruhe zurück. Einesteils mag dies mit der Stärke des Affekts zusammenhängen, obgleich diese Stärke auch wieder zusammenhängt mit dem Inhalt und Zustande der noch normalen Psyche. Denn das gleiche, was das eine Individuum affektiv stark erregt, geht an einen anderen Individuum spurlos vorüber. Nicht die Stärke des Affekts erklärt also genügend seine genannte außerordentlich starke Nachwirkung. In dieser überaus starken sekundären Funktion möchte ich nun das Besondere und Pathologische solcher Fälle sehen. Wundert sich doch unsere Patientin nota bene darüber, daß jetzt nach siebzehn Jahren das Bild ihres ersten Verlobten ihr niemals ins Gedächtnis kommt. Was denn aber diese starke sekundäre 
Funktion verursacht, bleibt auch mir dunkel; wir werden zu ihrer Erklärung wohl auf das Studium und die nähere Analyse der psychischen Anlage verwiesen und dies stellt ein Problem dar, dessen Lösung in der Zukunft liegt. Zur Erklärung der Tatsache aber, daß es unter der Einwirkung der andauernden Nachwirkung eines starken Affekts zum Ausbruch eines psychotischen Zustandes kommen kann, will ich noch darauf hinweisen, daßs solche andauernde starke Affekte auch für den physischen Haushalt des Körpers nicht gleichgültig sind; im Gegenteil führen sie physisch-chemische Veränderungen herbei, welche sich in starker Abmagerung und auffallender Schwächung des Körpers kundgeben können, sei es auch zum Teil wenigstens infolge einer zu geringen Nahrungsaufnahme und einer hartnäckigen Schlaflosigkeit.

Ich komme also zum Resultat, daß es unter der Einwirkung eines starken Affekts nicht nur zum Ausbruch einer heilbaren akuten Psychose des psychopathischen Typus (Birnbaum, Bonhoeffer u. a.) kommen kann, daß aber die andauernde Nachwirkung eines mehr oder weniger starken Affekts bei bestimmter, uns bis auf heute unbekannter psychischer Anlage eine chronische, unheilbare Psychose herbeiführen kann, und zwar andrer Art und andren Verlaufs als die Paranoia oder die circumscripte Autopsychose auf dem Boden der überwertigen Idee Wernickes. Weshalb in solchen Fällen Wahnideen, Halluzinationen usw., kurz von der Norm her nicht bekannte psychische Phänomene auftreten können, bleibt uns dabei in letzter Instanz zwar auch unbekannt; die Annahme eines psychisch-physischen Prozesses im Sinne Jaspers ist dazu aber unnötig. -

Diese Auffassung des Auftretens einer chronischen unheilbaren Psychose infolge der andauernden Einwirkung eines affektiven $\mathrm{Zu}$ standes bei einer besonderen Persönlichkeit schließt sich nicht der Auffassung des krankmachenden Einflusses des nicht-abreagierten und ins Unterbewußtsein verdrängten Affekts im Sinne Freuds an. Unsere Patientin reagiert gleichsam täglich, auch schon vor dem Ausbruch ihrer Krankheit, ab; sie sucht eine Lösung, aber keine Verdrängung des bewußten Konflikts. 